\title{
Towards an interoperable healthcare information infrastructure - working from the bottom up
}

\author{
D Ingram, D Kalra, T Austin, M W Darlison, B Modell and D Patterson
}

Historically, the healthcare system has not made effective use of information technology. On the face of things, it would seem to provide a natural and richly varied domain in which to target benefit from IT solutions. But history shows that it is one of the most difficult domains in which to bring them to fruition. This paper provides an overview of the changing context and information requirements of healthcare that help to explain these characteristics.

First and foremost, the disciplines and professions that healthcare encompasses have immense complexity and diversity to deal with, in structuring knowledge about what medicine and healthcare are, how they function, and what differentiates good practice and good performance. The need to maintain macro-economic stability of the health service, faced with this and many other uncertainties, means that management bottom lines predominate over choices and decisions that have to be made within everyday individual patient services. Individual practice and care, the bedrock of healthcare, is, for this and other reasons, more and more subject to professional and managerial control and regulation.

One characteristic of organisations shown to be good at making effective use of IT is their capacity to devolve decisions within the organisation to where they can be best made, for the purpose of meeting their customers' needs. IT should, in this context, contribute as an enabler and not as an enforcer of good information services. The information infrastructure must work effectively, both top down and bottom up, to accommodate these countervailing pressures. This issue is explored in the context of infrastructure to support electronic health records.

Because of the diverse and changing requirements of the huge healthcare sector, and the need to sustain health records over many decades, standardised systems must concentrate on doing the easier things well and as simply as possible, while accommodating immense diversity of requirements and practice. The manner in which the healthcare information infrastructure can be formulated and implemented to meet useful practical goals is explored, in the context of two case studies of research in CHIME at UCL and their user communities.

Healthcare has severe problems both as a provider of information and as a purchaser of information systems. This has an impact on both its customer and its supplier relationships. Healthcare needs to become a better purchaser, more aware and realistic about what technology can and cannot do and where research is needed. Industry needs a greater awareness of the complexity of the healthcare domain, and the subtle ways in which information is part of the basic contract between healthcare professionals and patients, and the trust and understanding that must exist between them. It is an ideal domain for deeper collaboration between academic institutions and industry.

\section{Introduction}

Healthcare policies throughout the world emphasise the importance of effective information services, in enabling and supporting the delivery of healthcare services that people need and value. Numerous studies over many decades have revealed systemic problems in these information services, and their adverse impact on the quality and cost of care. There has been recurring and expensive failure to understand and resolve the underlying issues, satisfactorily, sustainably and at scale.

Most successful innovation in healthcare information systems, internationally, has had its roots in relatively small-scale, practically focused work, based near to the ground and promoted by credible service champions, in local and well-defined clinical communities. But such innovation has not often proved capable of 
dissemination more widely, from the bottom up, in large part because it lacked a common, enabling and sustained parent information infrastructure within which to evolve and grow. Creation of such an infrastructure clearly requires significant ownership, planning, investment and capability to be organised and applied, from the top down.

The term 'healthcare information infrastructure' implies a coherent and comprehensive set of information systems and services, necessary and sufficient to support the goals of healthcare services and their governance. The scale and complexity of the challenge it poses, the discipline of underpinning standards that it requires, and the depth and range of new partnerships that must be forged to tackle it, place this endeavour in a class of its own. Different national healthcare systems may require and expect different elements of supporting information infrastructure, but much will be in common, supported by industries working across national boundaries.

Some elements of healthcare information infrastructure, such as computer network services, are now generically applicable within and beyond healthcare and are supported by mature and successful industries. Hospital patient administration systems, general practice systems, and clinical applications catering to limited and circumscribed domains, have long been available as products and services, customisable to the needs of specific healthcare groups or institutions, albeit that they often still struggle to provide the resilience and adaptability that changing clinical services require.

Some ambitions, such as the much discussed generic personal electronic health record, remain aspirations, and are only slowly evolving a common understanding of the requirements to be met and a mature evidence base on which to evolve the clinical and technical standards applicable. The governmental, professional and industrial collaborations through which they must be owned and evolved, experimentally, in relevant practical contexts, are not yet adequate to the task.

A key challenge faced in the context of determined national attempts, today, to create and introduce a standardised national healthcare information infrastructure, is how to scale up the successes of local, clinical and service-driven innovations, to serve similar community needs, further afield, and yet still retain effective opportunity for local and specialist diversity of information systems, since that, too, is needed.

This paper explores the wider context of this longstanding problem, with special reference to electronic health records and international progress towards a valid and robust technical and clinical architecture for their interoperability. It describes two case studies of initiatives, led from the Centre for Health Informatics and Multiprofessional Education (CHIME) at UCL, working at local, national and international levels, which have demonstrated success in working from the bottom up. These initiatives are grounded in everyday clinical practice, which is the right place to explore a new informatics discipline applicable to innovation in the field. Only there is it possible to develop and implement innovative systems and approaches, working iteratively and experimentally, in the context of feedback from experience, and thereby also advancing the underlying discipline.

A key requirement and feature of the work exemplified by these case studies has been the strong and capable working communities developed, involving partnership among academic centres of excellence, health services, health professions, patient groups, and industry and standards bodies, nationally and internationally. The quality and staying power of these partnerships has been crucial to creating and maintaining momentum and progress.

One such community is the openEHR Foundation, a not-for-profit open-source software foundation established at UCL, four years ago, with former team members now based in Australia. With now approaching 80000 references on the World Wide Web, openEHR has quickly grown its membership across 66 countries. Another such community is the APoGI network, dealing with informatics resources to manage and disseminate information for patients about inherited disposition to disease, with the particular example of haemoglobin gene variants. Both these communities are dedicated to sustained efforts to transform and support the capacity of patients, clinicians and health services to use electronic health records and clinical information resources effectively, in a manner which seeks to address the flexibility and diversity of current healthcare needs and to anticipate challenges of the future. Each develops and maintains a very extensive set of resources based on its research, published on the Web from CHIME, and visited many hundreds of times a day, from all over the world.

\section{Healthcare and informatics - co- evolving contexts}

Healthcare services in developed countries have evolved rapidly in scope and increased considerably in cost, over recent decades. Scientific, technological and clinical advances have transformed the scope and quality of clinical interventions and more of these are, in principle, capable of being provided from community-based 
services, delivered at or near to people's homes, or from mobile devices carried around by them, through the day. These trends have necessitated wide-ranging changes in the professions and organisations delivering health services, and in the management and governance of those services, throughout the healthcare system. A further factor influencing organisational change is the correspondence, in many cases, between the health and social care needs of patients and of their carers and families.

Knowledge and awareness of social and demographic determinants of health and patterns of disease, in increasingly mobile and ageing populations, have developed considerably. Government healthcare services are now under more intense external scrutiny than ever before, transforming and transformed by changing public, patient and carer attitudes, roles and expectations. Private, charitable and voluntary sector services make increasingly important and influential contributions to healthcare and policy. Patient safety, professional staff registration and deployment, and the confidential handling of personal health data have moved into an era of more exacting and prescriptive legislation and governance.

These trends have, over the same decades, paralleled spectacular evolution in basic information and communication technologies. Information has become an extremely pervasive concept, perhaps the most pervasive of our age. In a Ciba Foundation lecture in 1990, Zimmerli [1] described the transforming power of information technology. He called it '... the one and only 'horizontal technology', a technology that pervades each and every part of social life and all the other technologies, as well'. Similar words could equally well be used to describe modern medicine.

The co-evolution of informatics and medicine over the past 50 years has both reflected and influenced immense change in the requirements, scientific and technological foundations, delivery and accountability of healthcare services. Research endeavours within the healthcare domain have stimulated progress in informatics concepts and methods. For example, a highly successful, widely disseminated, and innovative approach to database technology of its time, MUMPS, had its origins in the work of an outstanding clinical pioneer, Octo Barnett, at Massachusetts General Hospital, in Boston. Correspondingly, practical efforts to develop information systems to support healthcare have prompted questions about clinical requirements, methods, quality and cost-effectiveness of services. This kind of encounter can be uncomfortable as it may sometimes reveal that we are not as capable as we might believe of articulating, in the rigorous manner that computerisation requires, what the discipline of medicine is and how it functions. That may, in turn, reveal that what we aspire to achieve is beyond current knowledge and capacity, which can also be quite an uncomfortable realisation.

Nonetheless, over time, the pushes and pulls of this co-evolution have helped to clarify and make more explicit aspects of medicine as a discipline which had not previously been confronted by the need to be clear and consistent about information and professional standards. The progress of common, computable domain vocabularies and terminologies, in the quest for valid and reliable means of coding and classifying diseases, treatments, care processes and outcomes is a good example of this kind of interaction. Through endeavours such as these, that bridge healthcare and informatics, the economic implications of healthcare's dependencies on quality of information have become ever clearer. Discipline underpinning the application of informatics concepts and methods to healthcare has evolved in a somewhat haphazard fashion, in various phases. The term 'medical computing' was adopted by the Medical Physics community, in the 1960s, when applying computers in its professional roles, for example in clinical measurement and imaging, planning and control of radiotherapy treatments and support for the administration of laboratory and other hospital services. The emergence, thereafter, of 'medical informatics', in the 1970s, signalled wider academic interest and enquiry about the relevance and application of computer science to knowledge management, record keeping and decision-making problems arising in the medical domain, in the diagnosis and treatment of patients.

'Health informatics' followed a decade later as the organisational and multi-professional scope and interrelationships of quality, information and governance issues, within the wider healthcare system, became clearer. 'Bioinformatics' established itself as a basic discipline of biology, quickly in molecular biology and, more slowly, in cell and systems biology. Information for consumers about the increasing range of health products and services available in the High Street and information about public health, have widened the health-related informatics domain still further. Health informatics and bioinformatics are now, increasingly, seen as interconnecting domains. For example, cohort studies of the genetic determinants of health require linkage between laboratory DNA analyses and personal health records. Driven by the need for consistency and coherence in the linkage of data and models from across these diverse areas, the prospects for a unified biomedical or bio-health informatics academic discipline are being explored [2].

Such a discipline is needed to underpin progress towards a more unified information infrastructure for 
both biomedical research and healthcare delivery. Healthcare information infrastructure is led, internationally, by the Connecting for Health Agency in the UK, Infoways in Canada, HCII in the USA and EHTA in Australia. Its development is paralleled, in different scientific communities, by the emergence of computational grids and data repositories, as part of international e-Science infrastructure initiatives. These are, for example, linking large-scale instruments such as the LHC at CERN or the Diamond synchrotron at Harwell, with the scientific communities across the world that use them. The data sharing initiative of the Medical Research Council (MRC) is exploring common infrastructure to support long-term cohort studies, linked with similar endeavour in the economic and social sciences.

A unified strategy embracing personal electronic health records and information management within the wider context of the needs of healthcare organisations and services is now generally recognised to be of central importance. However, healthcare is still learning how to achieve and sustain balance and interoperability of information systems dedicated across a wide spectrum, from information about the care of individual patients, as individuals (i.e. centred on their individual needs), through the particular information needs of diverse clinical specialisms, to health, performance and wellbeing statistics at a population level (i.e. centred on description and characterisation of groups). Requirements, potential solutions and wide-scale implementations continue to evolve, tuned to the diverse scales, functionalities and levels of performance required at these different levels of information systems.

The advent of portable information appliances and information utilities, available over the Internet, will progressively support pervasive and open sharing of information among citizens and healthcare professionals and enterprises. These flexible utilities will support a greater diversity in the delivery of services, requiring reappraisal of the organisational and ethicolegal boundaries, scope and regulation of the professions and services involved.

\section{Information for health - a wicked problem}

It is not hard to find contemporary evidence of medicine and healthcare producing, working with or overloaded by poor quality records [3]. The problem is not new. An early pioneer concerned with the poor quality of records and its impact on quality of care wrote as follows.

'In attempting to arrive at the truth, I have applied everywhere for information but in scarcely an instance have I been able to obtain hospital records fit for any purpose of comparison. If they could be obtained, they would enable us to decide many other questions besides the one alluded to. They would show subscribers how their money was being spent, what amount of good was really being done with it or whether the money was not doing mischief rather than good.'

Florence Nightingale, 1863

One hundred and forty years after these observations, a recent study of patients' experiences of health services across leading world economies [4] has revealed similar widespread systemic failure, today, relating to quality of information. Why is the problem so intractable and what can be done about it? Rittel and Webber [5] characterised the class of what they termed 'wicked problems':

- which are never completely solved,

- which do not have right answers, but do require good enough approaches,

- for which work towards the solution clarifies and revises understanding and definition of the problem,

- for which solutions require change in behaviour of people and groups involved,

- where there is no clear ownership of the problem, or possibility of permission to experiment towards finding a solution, or acceptance of failure in any who attempt to tackle it, or role or right of any group to judge solutions,

- where the manner in which the problem is tackled is as important as how it is tackled.

There may be another attribute - that they appear fractal in nature, giving rise to the same sorts of difficulty, confusion and distress, at whatever level or from whatever viewpoint they are observed and experienced.

Healthcare information exhibits most if not all of these attributes. Four successive national attempts, over four decades, to tame it in the NHS, motivated at successively higher levels of NHS management and Government, launched with successively higher aspirations and expectations, have dissolved into the sand after about seven years, as have many initiatives in regional or more local contexts.

Increasing pervasiveness of information technology gives rise to new problems of interoperability, confidentiality, and governance, as well as to problems of rapid technological obsolescence of component systems. Solutions to these problems are complex, 
reflecting different kinds of, not necessarily compatible, external constraints - available technology, legislation, funding, capability, capacity, standards. Healthcare has unique requirements in terms of the complexity and longevity of the records it must keep. It will need new kinds of standards for these if it is to be capable of sustaining benefit from its investments in information infrastructure. It still exists within a poorly defined framework of data standards, information systems and integration, and lacks a satisfactory approach to quality management. That is a clear and compelling rationale for a national approach, but one based on research evidence.

Within healthcare circles, historically, fitness for purpose of information systems has generally been seen as 'someone else's problem', until perhaps a decade or so, ago, when it rapidly became seen as almost everyone's problem! Unfortunately, problems that are unperceived and problems that are perceived everywhere can be almost equally incapacitating, suffering from either no owners, or far too many. The following reports have highlighted different issues and perspectives.

\section{- UK Audit Commission 1995}

A 1995 report of the UK Audit Commission [6] showed that in a typical UK acute hospital, $15 \%$ of money and $25 \%$ of clinical staff time was spent on information management tasks.

\section{- European Healthcare Trends 1995}

In his wide-ranging report on healthcare management trends across Europe, Rosleff succinctly expressed the goals of healthcare management as '... to maximise health gain of a community, within limited resources, by appropriate range and level of services, by monitoring on a case-by-case basis, to continuously improve care, to meet national targets for health and individual health needs' [7]. $\mathrm{He}$ highlighted the difference between policy, disease, healthcare system and patient perspectives on information, and the need for enhanced information infrastructure.

\section{- Learning from Bristol 2001}

In his report on the national enquiry into high death rates in children's cardiac surgery services at the Bristol Royal Infirmary, Kennedy highlighted underlying information problems, saying that '... information is the basic building block of any system of standards and quality' [8].

To relate information management to quality of healthcare, clarity and coherence is needed in what is measured, recorded and communicated, how, in what context, why and under what governance.

Despite this seemingly self-evident proposition, understanding of the conceptual foundations of evidence, decision-making and information management, such as in health records, has not played a vital and explicit role in clinical professional education and has, historically, been strongly resisted as a proper or important area of discourse. Also, the concepts and implementations of available computerised health information systems still have many limitations and inadequacies, and are often opaque in terms of how they have been designed, implemented and validated and what knowledge they embody. This makes meaningful education about them and their safe and effective use quite difficult.

Lessons learned about quality management in other sectors, such as the airline industry have led to new ways of thinking about safe operation of complex systems, highly relevant to contemporary concerns about the safe delivery of healthcare. The management of patient safety and clinical risk is seen to be a complex, whole-system function, involving organisational structures and procedures, professional practices, skills and competencies, appropriate information and communications systems, and a culture and practice of teamwork and self-evaluation, validated through external regulation. Experience of critical incident reporting and analysis, development, implementation and evaluation of practice guidelines and prompting procedures, and handling of litigation, all have lessons for clinical records and healthcare information infrastructure.

The issues highlighted in these reports pervade the healthcare enterprise, and the time and cost associated with them are immense, recently estimated at \$80billion per annum for the US health economy alone [9]. It is surprising, therefore, that the response is still, so often, to seek to spend more money and collect more information. The core issues and grand challenges are systemic and go to the heart of how medicine thinks about and communicates about itself. There is a great need for sustained programmes of basic research to provide grounding for healthcare information infrastructure, and thereby to underpin systems that enable and support good practice. Valid research must be framed from practice as well as transferred into practice. This research is arguably as important, in terms of patient safety and economic impact, as pharmacological research that provides the scientific grounding of clinical therapeutics. It is said that it takes 17 years to take a new drug from the initial stage of observation of a novel pharmacological effect to a fully 
evaluated and delivered product, integral with good clinical practice and education.

The lesson of experience is that where such issues are not resolved in terms of clinical requirement and method, service management and implementable technology, the technology, the most brittle domain of the three, and those who provide and support it, will indeed break and therefore become the problem.

It is not difficult to find examples of high-level policy documents about transformation of healthcare through IT [10]. It is noteworthy, though, how little the aspirational rhetoric has changed in four decades [11]. With hindsight, one can see how the wrong assumptions people from different disciplines and with different roles easily make about each other's needs and contributions can be very wasteful and damaging.

After 20 years of effort, there is now much greater international consensus on clinically focused requirements, specifications and technologies, to guide appropriate, empirically grounded standards-making processes, and thereby enable a strong, coherent product market-place.

Many countries are grappling with the generic, both grand and wicked, challenge of healthcare information infrastructure. The holy grail is the electronic health record, arguably only soluble from a combination of experiments in practical implementation, from the bottom up, linked with and informing standards-making processes and a generic information infrastructure, from the top down.

\section{Electronic health records - a key policy objective}

At the heart of clinical practice, and therefore at the centre of the impact of healthcare information infrastructure developments at ground level, are health records. These must be, and remain, faithful to the processes and meanings they document, when:

- acquiring knowledge about specific patients (questioning, observation, measurement),

- interpreting and reasoning critically with this knowledge (in the context of many relevant scientific disciplines and technologies, personal knowledge and experience, population health needs),

- deciding upon, carrying through, monitoring, evaluating interventions (in partnership with patients, carers, teams, organisations).
The practical exigencies of clinical service delivery and healthcare management require personal clinical information to be captured, stored, processed and communicated, ensuring that this is done in an appropriate and timely manner, with consent, rigorously, confidentially and efficiently. An equally important goal is to assist, inform and guide patients and citizens in the choices and actions they make and take, about their health and healthcare needs and options. To meet such goals, health record systems must be developed and operated against clear and practical clinical, technical and managerial, as well as ethico-legal requirements and standards.

Clinical information must be aggregated and analysed at individual, group and population levels, in support of service management and also of research and development. The domain has proved extremely hard to standardise. Common standards are needed, but these cannot be fixed and absolute, and an empirical standards-making process is required in which all parties develop trust and a sense of ownership. The diversity of health and social care activities, and their supporting industries and organisations, inevitably encompasses multiple perspectives on the needs and priorities, delivery and accountability of healthcare. Hence, effective and robust standards processes, though vital, have proved very hard and timeconsuming to create and sustain.

Perhaps not surprisingly, therefore, there is no topic more central, nor more fought over, in the evolution of health information infrastructure and standards, than the electronic health record. Implemented and operational electronic health records have been commonplace for several decades. But a generic and evolving, standardised approach, meeting the needs of diverse clinical practice, has proved an extraordinarily taxing challenge, at national and international levels. The Workplan of Framework 4 of the EU in 1989 set out the aim to:

'... unify European activities by providing the means for efficient communication of medical records and knowledge so that these may be understood and compatible, thereby permitting the integration of health information systems.'

This goal has led to a huge amount of $R \& D$ and also time and debate in Europe (Centre for European Normalisation, CEN), the system suppliers community (HL7), and the International Standards Organisation. In CEN and ISO, it is currently moving slowly through successive draft standards towards common understanding - now, fortunately, based more on shared goals and empirical implementation experience. The connectivity of the record is highlighted in Fig 1, taken from Ingram et al [12], formulated fifteen years 


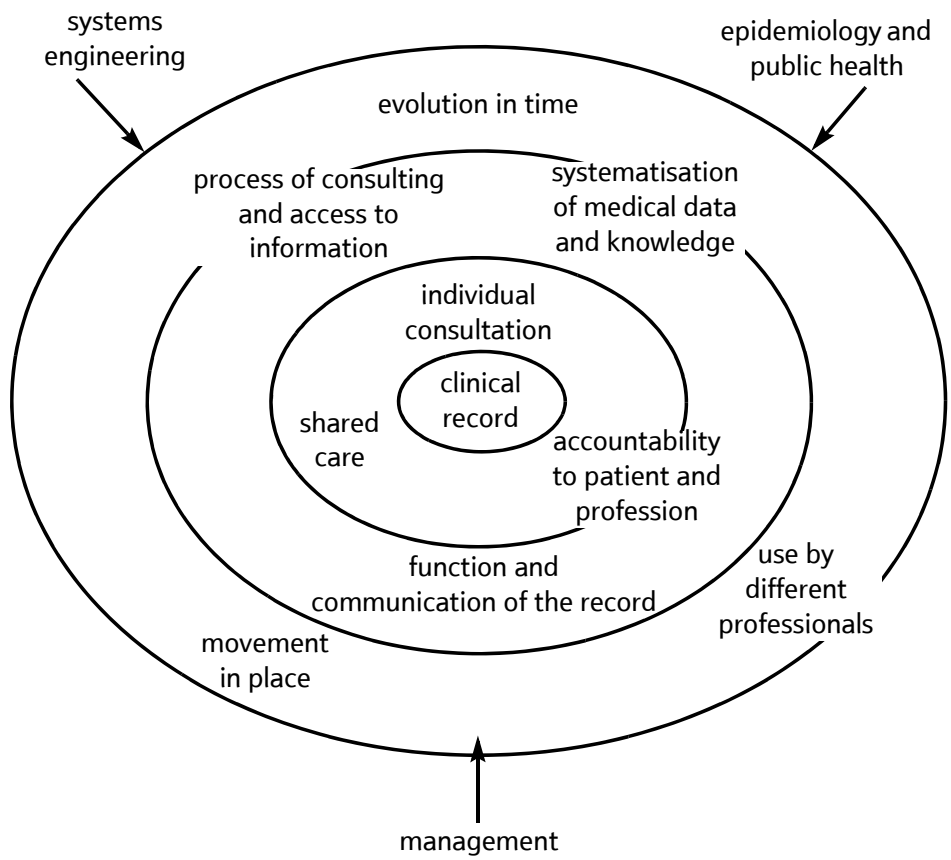

Fig 1 The domain of health record requirements formalised within the EU GEHR project [12].

ago at the outset of the founding research of the CHIME team on health record architecture. This research has been taken forward in successive projects (GEHR, Synapses, Synex, Medicate, 6WINIT) of the EU Framework Programme, in the UK eScience programme (CLEF, CLEF-Services), in the evolving healthcare record standards of CEN (TC/251) and ISO (TC/315), and in the openEHR Foundation. It places the record within its personal, clinical, technical and management requirements and context. It builds a rigorous formalism, generically adaptable to accommodate local customisation and evolution in time of so called clinical data archetypes, matched to carefully scoped and empirically validated terminologies, built to a common formalism and integrated around a common reference information model $[13,14]$.

If clinical meaning and context is to be expressed and communicated, confidentially, in electronic records, some such approach is needed. Professionals and patients must be helped and enabled to become much more involved, able to take responsibility for those areas of the enterprise that intimately relate to their roles in harmonising and advancing the quality of their practice and care. To begin to achieve this we require a new level of discipline and transparency of information systems and of the services they provide and support. The 'information utility' concept looks to a world of standardised services which better protects investments in information infrastructure, provides choice and customisation potential, and moves forward with evolving clinical need while maintaining technical viability.
When reviewing quality of care, there is a fine granularity of clinical information, including personal narratives of events, through which the meaning and context of events described are communicated in records and other communications. Such information may prove crucial in understanding and seeking to avoid critical incidents. It may not be possible to summarise or abstract this through codes or classifications while still retaining the relevant clinical context and meaning. A key issue is the extent to which electronic records must be capable of capturing this rich detail, to inform events or analysis elsewhere within the overall healthcare delivery system.

Health information products and services represent a huge and growing business, but health informatics has struggled to create a sustainable professional identity and academic priority. The industry requires a disciplined and professional approach from healthcare services purchasing its products and very often in the past has not had one.

Going to scale challenges leadership and organisational skills and competencies as well as the development, availability and sustainability of appropriate products and services from the industry. In building a more successful strategy for going to scale, practical implementation experience, knowing, and learning the lessons, must be given greater weight. If we push forward too quickly, many current software vendors will not be able to sustain their products, beset by accumulated entropy in hurriedly patched-up systems, crying out for unaffordable fundamental redesign. 
Institutional investments in data, tightly coupled to such systems, will be at risk. However, we must face the fundamental changes in clinical information infrastructure implied by changing clinical needs, changing patient expectations and changing clinical service delivery. It is unlikely that this can be tidily managed without some, perhaps quite considerable, losses in the short term, in the interests of longer-term gains.

That said, the opportunities to make a transforming change, as the NHS now intends, are considerable, given the willingness at government level to make the necessary investments, in time, effort and money. The challenges can now be tackled with the benefit of experience from much pioneering innovation.

So what has academia to offer to these powerful programmes. In the remainder of this paper we will introduce two areas of current research in CHIME and describe the motivation and contribution of the work.

\section{CHIME}

CHIME (the Centre for Health Informatics and Multiprofessional Education) [15] works at the intersection of clinical method, information technology and healthcare delivery and clinical research.

It is situated at the Whittington Hospital Campus of the Medical School of UCL, at Archway in North Central London. It brings together a wide-ranging research programme in health informatics and associated health service change and development, with undergraduate and post-graduate educational programmes covering topics in information, quality and governance for healthcare.

CHIME is founded on the principle that close contact with real healthcare needs and services must be the foundation of its research and teaching. We put this into practical effect. We have a long track record in national (Research Councils, DH) and international (EU Framework Programme) research as well as in international standards activities for the field (CEN, ISO, HL7, openEHR). On the other hand, our work is grounded in innovation and implementation of practical information systems and services required for healthcare and in the quality and governance frameworks within which these operate, for example:

- electronic records - anticoagulant therapy, heart failure and chest pain management, linking hospital, High Street Pharmacy and PCTs/GP surgeries,

- $\quad$ knowledge management - international screening information resources about genetic variability and clinical implications of haemoglobin disorders,
- decision support - development and evaluation of methods for diagnostic image interpretation,

- organisational development - managing effective change.

CHIME offers part-time certificate, diploma and masters level courses in health informatics and risk management. Our student group is truly multiprofessional and drawn from across Europe and further afield. Many are seconded to, and funded on, the courses from full-time employment in the NHS. We are currently able to admit 20 students per annum to each programme and know, from our close contact with past students, that the qualifications are providing a springboard to rapid career advancement in the changing world of healthcare services that is emerging, internationally.

\section{Case study 1 - anticoagulant therapy}

A key collaboration of CHIME has been with the cardiology department of the Whittington NHS Trust and the services it provides in partnership with primary care and pharmacy in North Central London. The issues we focus on here are:

- $\quad$ providing a shared clinical record and supporting critical decisions about drug prescribing,

- an experimental domain in which to study the clinical governance required for a new devolved service linking hospital doctors, GPs, pharmacists and the patients themselves in the shared care of patients,

- exploration of whether this provides an extensible paradigm for flexible management of chronic disease,

- shareable terminology linked with record architecture,

- experimental development of a generic information architecture and systems to join up the records of clinical services and professions working together in a new service context,

- an internationally credible demonstrator and test bed for the evolving new CEN/ISO and openEHR standards for health record architecture.

The population of the part of inner city London in which CHIME and the Whittington are based comprises a wide ethnic mix which poses an increased specific health risk - deaths from heart disease are more common in people from the Indian subcontinent and deaths from stroke are more common in African and Caribbean people. The boroughs have relatively high levels of deprivation, proven to increase the risks of heart disease through smoking, hyper-tension, raised 
cholesterol, diabetes, physical inactivity and poor nutrition.

There is an increasing need for anticoagulant and stroke-prevention services due to the improved detection and management of patients with irregular heartbeat such as atrial fibrillation. Warfarin has been shown to reduce the likelihood of stroke in such patients. However, warfarin does need to be carefully controlled by regular blood testing in order to ensure that the risks of haemorrhage and of anticoagulantrelated death, are minimised and the benefits of anticoagulation are maximised. A hospital based service is no longer necessary for the delivery of the service to patients who need careful monitoring as a result of:

- the development of a reliable near-patient testing device - this permits the monitoring of anticoagulation closer to the patient,

- the CHIME electronic advisory and management system - this ensures that the quality of anticoagulant control can be monitored by site of service delivery, by dosing clinician or by patient, while also ensuring that the up-to-date clinical information is available electronically to the authenticated clinician wherever the patient attends.

The use of warfarin is complex and requires close collaboration between primary care and secondary care clinicians. The NHS litigation authority has reported that medication errors involving anticoagulants fall within the top ten causes of claims against NHS Trusts. Anticoagulants were included in the Department of Health report 'Making Medication Practice Safer' (2004) as high-risk medicines that require the implementation of additional safety controls. The National Patient Safety Agency (NPSA) has completed a comprehensive literature review and a risk assessment exercise, undertaken with a multidisciplinary group on the use of anticoagulants in the NHS (Consultation Paper, 2006).

The principles on which we base our distributed service (Fig 2) have a number of characteristics:

- the patient will receive the anticoagulant monitoring care in the most convenient and safe place for them,

- the anticoagulant practitioner will be demonstrably well trained, up to date and able to offer a highquality service,

- the clinical details of the patient and the advisory and management system will be available in a timely manner to the practitioner,

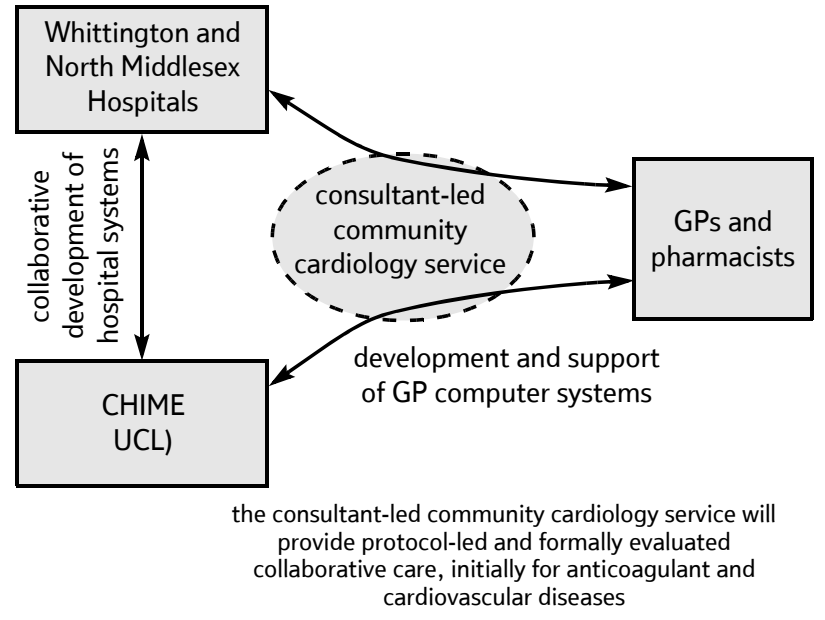

Fig 2 The new model of anticoagulant therapy service delivery at community level, made possible by the supporting health record and decision support system developed in CHIME.

- the Clinical Governance arrangements will be implemented and learning processes enhanced.

Our goal over the coming months is to move towards a distributed service where the patients have their anticoagulant control managed in the following settings:

Hospital setting for patients who are $10-20 \%$ complex

General Practice setting delivered by a practice nurse or the GP

Community Pharmacy

Patient self-testing

Patient self-management

$20-30 \%$

$30-40 \%$

$10-15 \%$

$10-15 \%$

The next phase of this service (Phase 5) in 2006 will build on the experience already gained at the Whittington. Fifteen years ago we developed our first electronic advisory and management system (Phase 1). Ten years ago we developed an outreach service in four Primary Care Centres (Phase 2). In January 2002 we developed a community pharmacy service in South Islington (Phase 3). In 2005 we started a service in Boots in Wood Green (Phase 4). After an initial successful pilot study a more developed service is now running with over 100 patients now being managed by community pharmacists. These pharmacists are also supplementary prescribers which has the merit of saving the patient a separate visit to their GP to obtain a prescription for warfarin. These distributed services in Primary Care or in the Community Pharmacy are supported by the hospital anticoagulant service (clinician and haematology laboratory).

The distributed services are supported by the CHIME developed electronic management and advisory system 
for anticoagulation and stroke prevention, which is one of a suite of modules using the same supporting electronic health record (EHR) (see Fig 3). The EHR server is sited at the Whittington Hospital and supported by the IM\&T Department. It is a secure, federated, Web-enabled system available to any authorised clinician wherever they are situated, whether this is in the hospital environment or in primary care. It draws on experience of over a decade of European Framework Programme and UK national eScience programme work. It is a unique, clinically useful, secure, robust, information management system, which has now been in clinical use for over five years. It plays a key role in the delivery of the Clinical Governance agenda. It is also suitable for other long-term conditions, and has already been applied to heart failure and chest pain management, where the overlaps with anticoagulant treatment are considerable.

The model of the patient self-testing and selfmanaging, in their home or elsewhere is now being developed as part of phase 5 of the collaboration. A number of suitable patients have been identified and they are keen to participate. The service will need to be set up as a pilot at the outset. This service will be evaluated before it 'rolls out' to a wider group. We intend to evaluate two educational packages in the next months and use the outcome of this to inform our future steps.

\subsection{Project support}

The CHIME research over a fifteen year period on the information architecture, record standards and clinical data repository demonstrators has come from MRC, EPSRC, EU and DTI research grants. Funding for

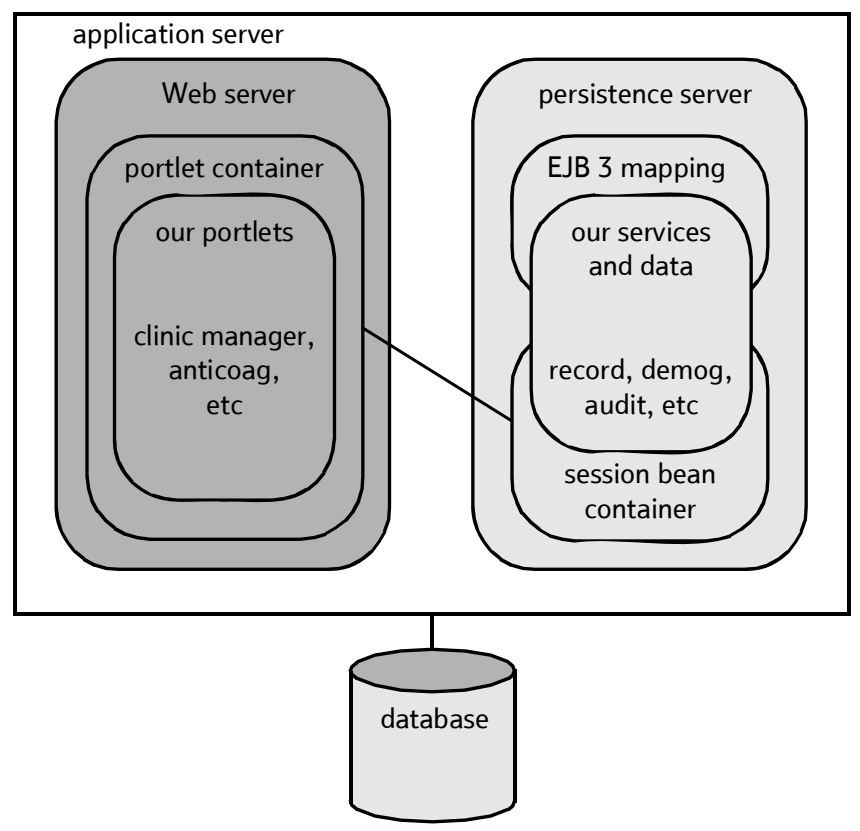

research focusing on Patient Groups has come from the SDO programme of $\mathrm{DH}$, and on operational research on service delivery in association with the DH CORU Unit. A technology demonstrator for IPv6 networking was funded by the EU.

\subsection{Case study - conclusions}

This case study provides a well-proven example of how local innovation in clinical service, partnering research teams in health informatics, has led to fundamental change in the delivery of clinical services, sustained, iteratively, in an experimental setting where all aspects have been evaluated and progressively refined through many generations of research projects. There has been successful evolution, too, in the capacity and preparedness of local cardiology, GP and pharmacy staff to participate. The cohort of patients managed in this way provides an ongoing, unique resource for studying and refining the interaction between the informatics innovation and service delivery, and is a leading international demonstrator of the state of the art in interoperable health records and decision support.

\section{Case study $2-$ the accessible publishing of genetic information (APoGI)}

The Whittington Hospital, working with the UCL Department of Primary Care and CHIME, has a longstanding reputation for its leading international roles in developments in care for patients with thalassaemia and the supporting screening, counselling and advisory services for patient and at-risk communities.

The work in CHIME since 1997, funded by grants from the Wellcome Trust and $\mathrm{DH}$, has focused on:
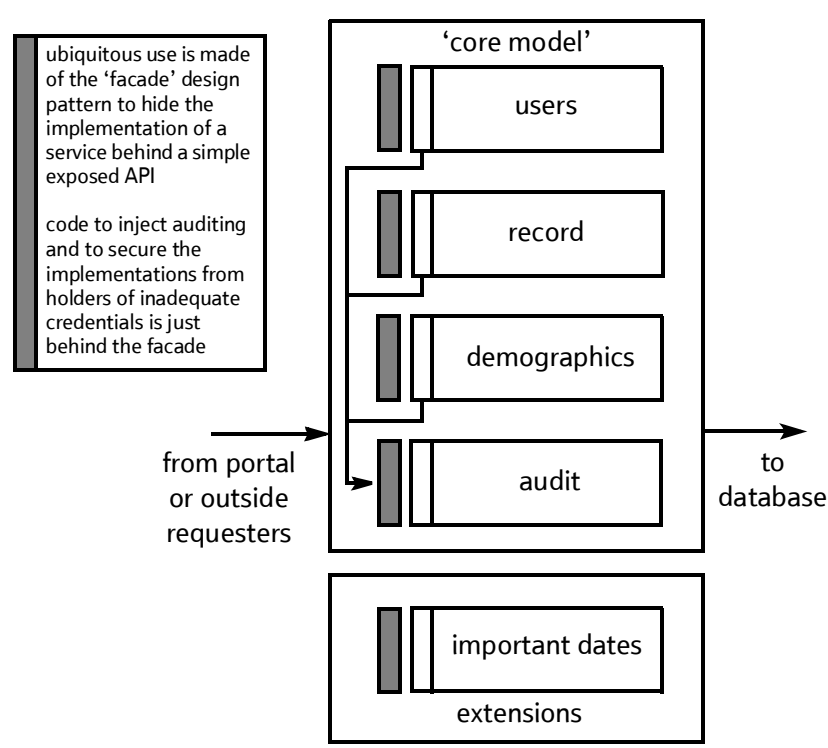

Fig 3 Federated record for anticoagulant therapy — schematic diagram of the software components. 
- experimental development of novel methods for managing, and making available to patient and professional communities, a large volume of information relevant to genetics advisory services about thalassaemia, in a flexible and sustainable way applicable to other similar disorders,

- working with patient and professional communities, locally, nationally and internationally, to be aware of and represent their perspectives and choices on these information services,

- building an information model to enable capture, curation and dissemination of new knowledge arising in screening for haemoglobin gene variants,

- supporting education and training for a national screening programme.

The team has been brought together from local clinical service leads, a national network of specialist clinical advisors, local and national patient groups, and an international network of associates and visiting academics to CHIME from all parts of the world. The work is recognised by the designation of Professor Modell's team in CHIME as a WHO Collaborating Centre for community control of inherited disorders.

\subsection{Clinical and scientific context of the work}

There is rapid progress in understanding the organisation of DNA into genes and chromosomes, and clinical molecular studies indicate that all human genes have hundreds of variants with important (though often different) health implications (see Figs 4 and 5). Techniques for screening DNA for thousands of variants are also in an advanced stage of development. In the coming decades these advances will coalesce into a body of knowledge giving unprecedented understanding of the biological basis of health and disease.

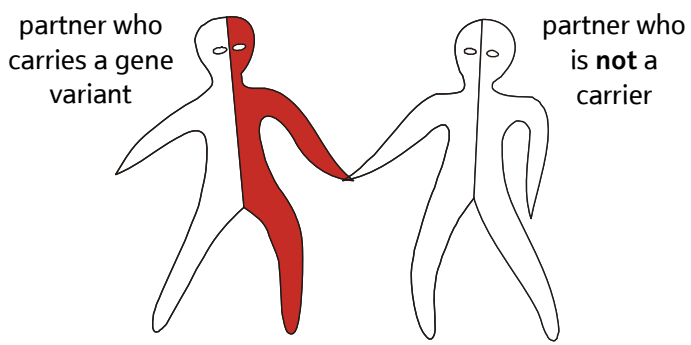

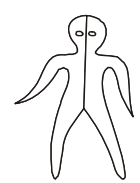

not a carrier

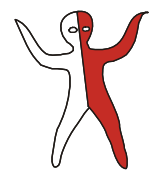

carrier of the gene variant

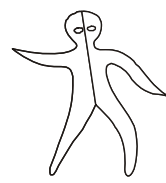

not a carrier

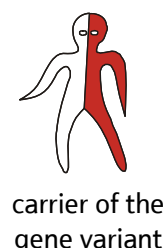

gene variant
Fig 4 A DNA variant may be present on only one chromosome, and may or may not have a perceptible clinical effect in a single dose.

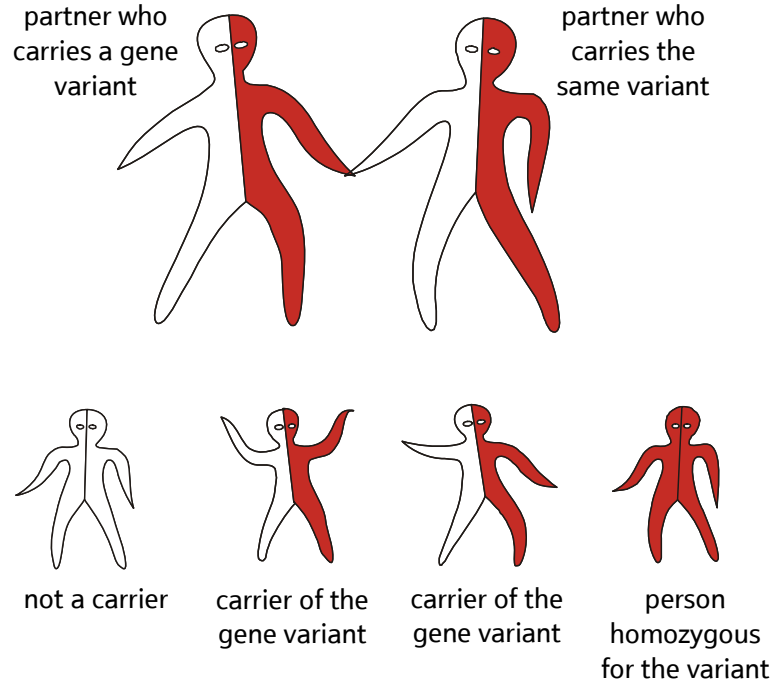

Fig 5 DNA variants are usually capable of being inherited in a double dose, which is highly likely to intensify any clinical effect they may have.

The new knowledge will offer previously unthought-of diagnostic opportunities, e.g. in screening for risk of single gene disorders, in identifying the genetic component in common diseases (cancer, cardiovascular disease, etc), and in therapeutic developments including pharmacogenetics and gene therapy. There will necessarily be pervasive changes throughout medicine.

In 1984, on the basis of a careful study of the challenges involved, WHO recognised the following generic problems - which are even clearer today than they were then:

- a public health perspective is lacking within medical genetics, with a reciprocal absence of medical genetics from the public health agenda,

- although information is the principal intervention in genetic counselling, there has been no systematic investigation of the requirements for acquisition, standardisation, processing and provision of genetic information for health workers and the public,

- there is no clear framework for promoting genetic approaches at the primary healthcare level,

- there is a lack of over-arching guiding principles and general concepts - in their absence, the diversity of the professional groups involved leads to fragmented thinking and difficulty in reaching consensus.

WHO therefore recommended that a science of community genetics should be developed to integrate genetic and public health approaches, define principles 
and basic concepts, and create methods and instruments, for example, for the following purposes:

- describing the epidemiology of genetic disorders in terms useful to clinicians and health service planners,

- cost analysis - describing the financial clinical and psychosocial burden of genetic disorders,

- audit - using registers to monitor service delivery and take-up,

- providing genetic information for health workers, patients and the public,

- developing healthcare infrastructures for delivering genetic testing and population screening.

We need to develop information systems both for making full information available to patients and the public, and for continuing consultation with the community on the uses they wish to make of the genetic information. The work also needs to address methods for obtaining information on patients' choices, and for providing information to health workers and the community.

\subsection{The haemoglobin disorders}

Haemoglobin is a relatively small protein molecule that is easy to obtain in an almost pure form because it is highly concentrated in red blood cells. As a result it has played a key role in molecular genetics research, firstly giving access to protein structure and later to the first direct studies on human DNA. The globin genes are presently the best understood of all human genes. There are currently around a thousand known haemoglobin gene variants. They fall into two groups those leading to under-production of a haemoglobin subunit cause a thalassaemia, those altering the structure of a subunit lead to an abnormal haemoglobin.

\subsection{Registers as genetic public health tools}

The UK thalassaemia (patient) register and the UK register of prenatal diagnoses for haemoglobin disorders have provided information on (almost) all affected children born, and all terminations of pregnancy for thalassaemia. Thus between them they provide data on the outcome of all known affected conceptions, summarised in Fig 6 - probably a unique picture, as it provides information on the outcome of almost all affected conceptions since thalassaemia first arrived in the UK in the late 1940s.

However, WHO had also suggested that audit should include both assessment of the effect of the service on the affected birth rate, and follow-up of the

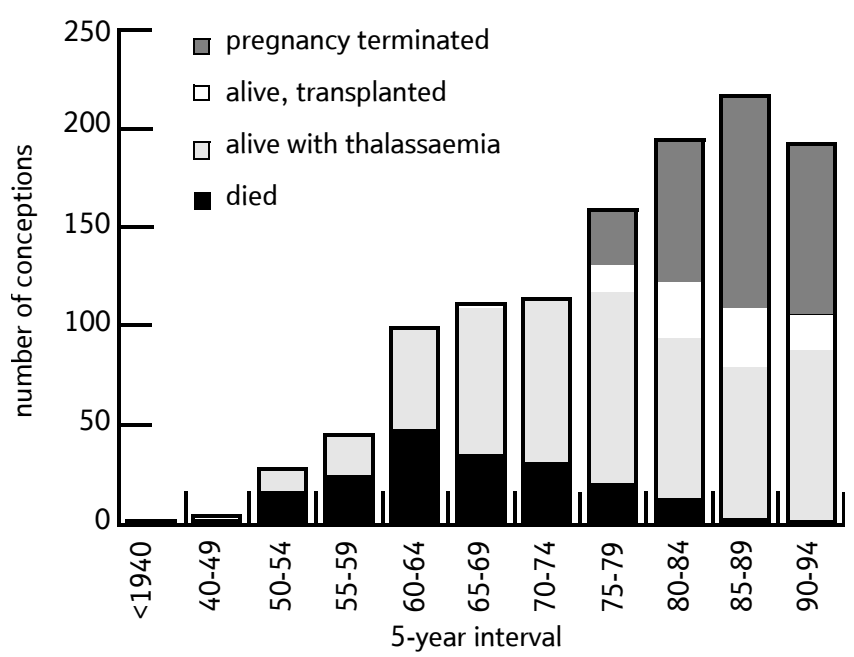

Fig 6 Outcomes over time for known affected pregnancies in the at-risk population.

affected infants born, to assess which births reflected informed choice on the part of the parents, and to locate problems in service delivery. In the UK, this recommendation was implemented in the National Confidential Enquiry into Genetic Counselling (CEGEN), co-ordinated by Professor Rodney Harris.

In the CEGEN thalassaemia module, informed choice was defined as the objective of the service, and the offer of prenatal diagnosis as the key indicator. The two thalassaemia registers were used to identify all couples with an affected pregnancy in 1990-94, and 88\% of the case notes were studied by the Confidential Enquiry team. The results showed that prenatal diagnosis had been offered in every pregnancy to only $50 \%$ of at-risk couples (with large regional variations).

Failure to detect risk was unavoidable in only $7 \%$ of these cases. A wide range of errors were identified, the most common being failure to test the woman or her partner, or to inform them, and including carrier misdiagnosis, clerical errors, and losing the information once it had been obtained. Most of the errors clearly reflected absence of a local screening policy and defined line of responsibility, and indeed a subsequent study showed that $65 \%$ of the 134 district health authorities in England did not have any written policy applying to the haemoglobin disorders.

\subsection{Financial cost of failing to deliver the service appropriately}

In certain countries, the early introduction of screening and prenatal diagnosis, and a strategy that ensures that all at-risk couples are informed prior to reproduction, has pinned the total annual cost of thalassaemia services at about $10 \%$ of the potential final level. In contrast, in the UK, inefficient service delivery related to absence of a national screening policy, and adoption of 
antenatal screening as the only approach, had allowed costs to reach $50 \%$ of the anticipated level in the absence of prevention. The findings also make a point that applies equally for cystic fibrosis screening - the ultimate economic benefits of introducing the service are steadily eroded with every year's delay in introducing it. Improved service delivery requires greater involvement of primary healthcare.

\subsection{Information requirements for supporting basic genetic services in primary healthcare}

Genetic counselling requires both genetic knowledge and counselling skills. Though many primary care workers have counselling skills, they lack access to the precise genetic information that would permit them to provide basic genetic counselling. Our hypothesis is that primary care workers will be able to meet the challenge of providing appropriate genetic advice if quality information is readily available to them through an Internet-based expert information system.

Fortunately in the UK there is a network of trained sickle cell and thalassaemia nurse-counsellors, who may be seen in some senses as a proxy for primary care teams. When consulted, the counsellors emphasised that information materials should be:

- diagnosis specific (i.e. genotype specific),

- equally available for rare and common diagnoses,

- designed to match each step in the screening cascade,

- graded to clients' range of education and desire for information,

- available in a range of languages.

It was relatively simple to produce a standardised carrier information leaflet, and a fuller carrier booklet, for each of these diagnoses. However, the most important genetic advice they contain is to ask the partner to have a carrier test. Consequently, once the partner has been tested, couples need diagnosisspecific leaflets with information on their particular genetic risk, if they have one. The most usual outcome is that the partner is not a carrier, but in fact they may carry any one of the 12 chosen mutations for which pilot materials were developed, or even a very rare mutation. Thus it proved necessary to produce 12 such leaflets for each carrier diagnosis. An example of an information sheet for an unusual combination is shown in Fig 7 .

\subsection{Information system for the haemoglobin disorders, in process of development}

The partners in this enterprise so far are the UCL Department of Primary Care and Population Sciences, the CHIME, the European Bioinformatics Institute, and the WHO non-communicable diseases programme. The
Implications for a child when one partner carries haemoglobin S (sickle cell) and the other carries haemoglobin O Arab

This couple could have a child with haemoglobin S/O Arab disorder
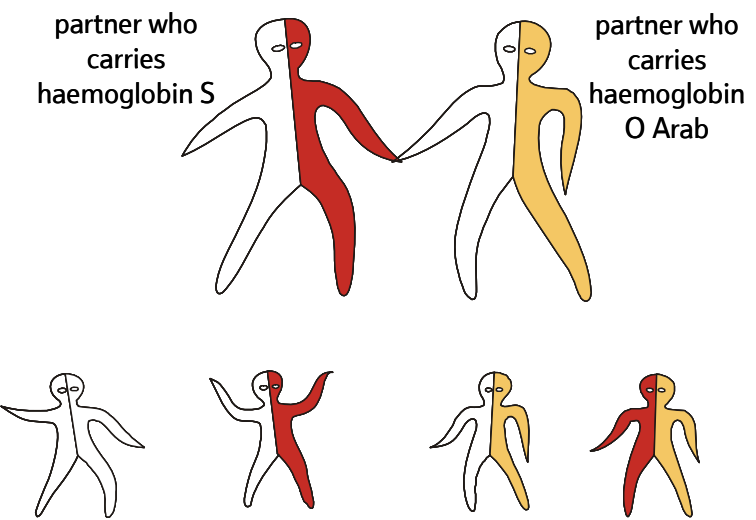

carrier of haemoglobin $\mathrm{S}$ haemoglobin $\mathrm{O}$ haemoglobin $\mathrm{S} / \mathrm{O}$ Arab Arab disorder

In each pregnancy, there are four possibilities:

- The child may not carry any haemoglobin disorder.

- The child may carry haemoglobin O Arab. This is harmless.

- The child may carry haemoglobin S. This is harmless.

- The child may inherit $\mathrm{Hb} \mathrm{O}$ Arab from one parent and $\mathrm{Hb} \mathrm{S}$ from the other, and have haemoglobin S/O Arab disorder.

Haemoglobin S/O Arab disorder is a sickle cell disorder. It usually causes anaemia, an increased risk of infections, and painful crises - unpredictable attacks of very severe pain, that can occur anywhere in the body and may last hours or days.

It is not possible to predict whether a particular couple could have children with mild,

moderate or severe sickle cell disorder.

It is possible to test a baby for Haemoglobin S/O Arab disorder early in pregnancy.

Fig 7 Example patient leaflet generated by the APoGl system.

product so far is the set of pilot information materials available at the Accessible Publishing of Genetic Information (APoGI) Web site (see Fig 8) [16].

Circumstances favoured the development of an information system for haemoglobin, because the necessary collaborations and datasets are already largely available as a result of the WHO recommendations. Our vision is that the haemoglobin prototype could be extended to other inherited conditions, because it is designed as a template that can be applied for any DNA-based (or equivalent) genetic diagnosis.

We believe this approach will prove widely applicable because it is urgently needed, and the advent of electronic health records will facilitate accumulation and banking of data. In addition, patient support associations are well aware of the need for quality information, and can mobilise extensive voluntary support from the public and among health workers.

\subsection{Case study - conclusions}

To achieve equitable delivery of genetic testing to the community, government policies, a focus on primary healthcare, and supporting information systems are 


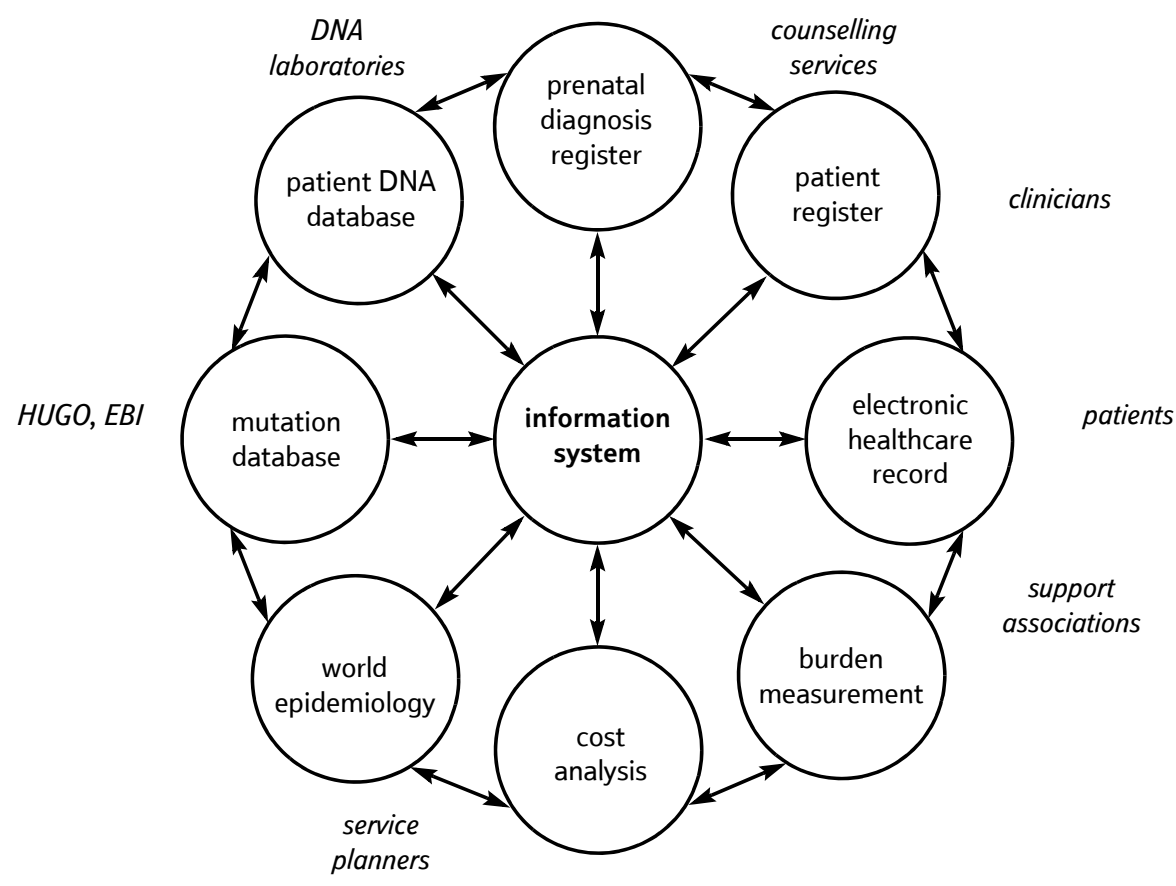

Fig 8 The range of information resources, ultimately linked with the electronic health record, developed to manage information for patients about haemoglobin disorders.

needed, including electronic healthcare records, linking basic scientists with service providers and the community. If such a system is developed, it only needs to be done once world-wide, and there is a clear need for international harmonisation.

\section{Future directions}

The engagement of industry in national healthcare infrastructure developments has necessarily focused on delivery of infrastructure planned and managed from the top down. As emphasised in this paper, that is not the direction in which sustainable innovation originates and disseminates in healthcare. Hence, attention needs now to focus on how these, both necessary but neither independently sufficient, top-down and bottom-up processes can be better harmonised because at present they tend to collide. Academia is good at bringing diverse expertise to bear on innovation; industry on building capacity and achieving the benefits of scale in affordable and sustainable services. Standards require a practical and pragmatic approach, built on an iterative and experimental approach to implementation of clinical systems at progressively greater scale. This requires academic, professional and industrial teams, working together, practically, but within a framework of governance which legitimises the experiments and the learning that are needed.

Future activities in CHIME along these lines include:

- building activities such as openEHR and APoGI as world-wide, clinically driven communities focused on achieving benefits for patients and practitioners, and building spin-off companies and industry collaborations for proven CHIME technologies and IPR,

- research on the broader information needs and service acceptance of the anticoagulant therapy service, for the communities of patients and professionals served,

- research on service change and dissemination of innovation,

- research and development for model clinical research data repositories (e.g. UCL/ICH Centre for Child Health Epidemiology, UKCRC Neurodegenerative diseases research network, MRC data sharing initiative, infectious diseases networks) based on the draft international standard health record architectures arising from research and implementation experience in $\mathrm{CHIME}$, and making these interoperable with key national clinical trials and longitudinal clinical research studies of the Research Councils and DH,

- ontology and development of clinical knowledge resources, interoperating with standardised health record architecture,

- development under contract of Web support for national education programme for screening services for sickle cell disease and thalassaemia.

Finally, Fig 9 indicates the scope of interaction between operational healthcare information and 


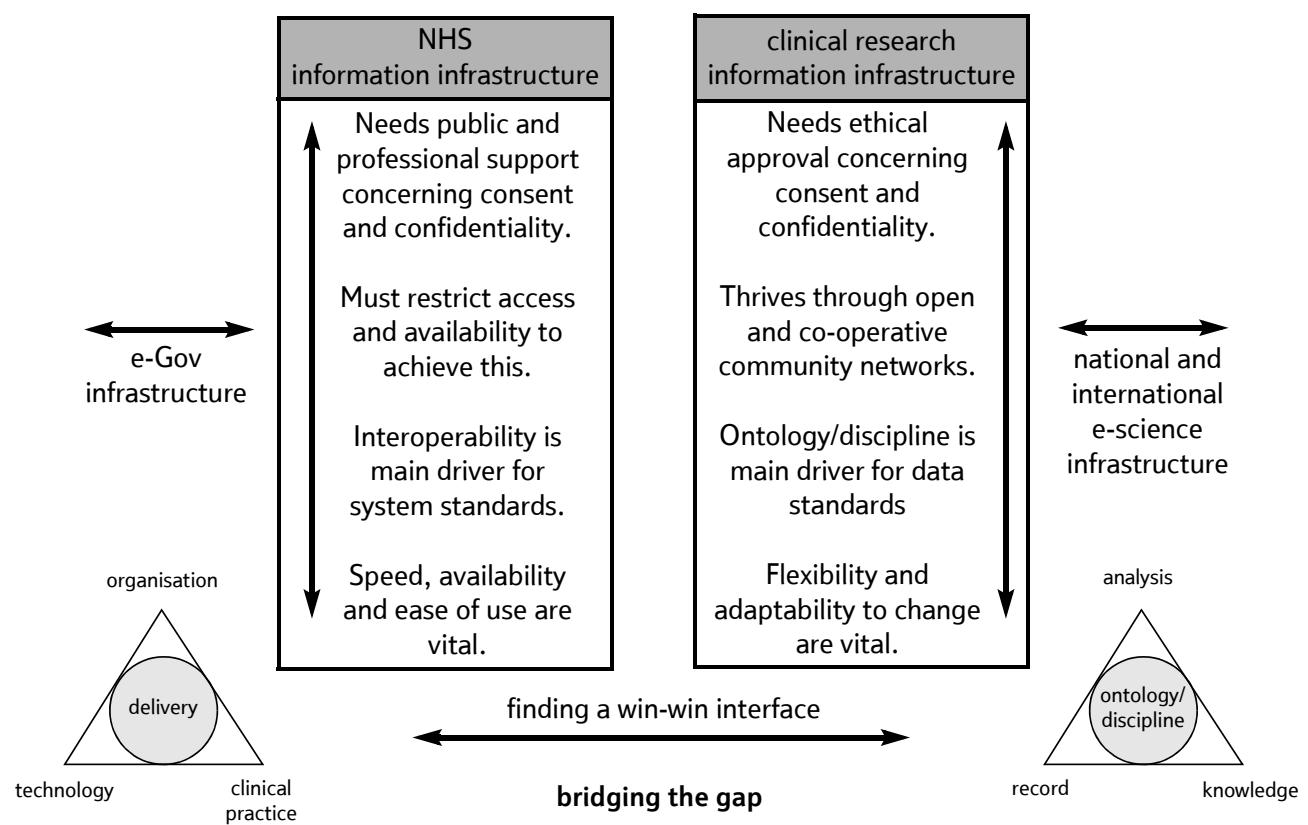

Fig 9 The competing pressures on healthcare and clinical research information infrastructure that need to be bridged.

research studies. It is of strategic importance for the UK that the NHS information infrastructure be developed consistently as a unique test-bed for enabling research where health records and laboratory and epidemiological datasets may be correlated and tracked, safely, over long periods of time.

\section{References}

1 Zimmerli W C: "Who has the right to know the genetic constitution of a particular person', in Human Genetic Information: Science, Law and Ethics, Ciba Foundation, London, UK, pp 93-110 (1990).

2 Johnson S P and Friedman R A: 'Bridging the gap between biological and clinical informatics in a graduate training programme', Journal of Biomedical Informatics (in Press) (2006).

3 'The Computer-Based Patient Record - an Essential Technology for Healthcare', Computer-based Patient Record, Institute of Medicine (1991)

4 Blendon R J, Schoen C, DesRoches C, Osborn R and Zapert K: 'Common Concerns Amid Diverse Systems: Health Care Experiences In Five Countries', Health Affairs, 22, No 3, pp 106121 (2003).

5 Rittel $\mathrm{H}$ J and Webber M M: 'Planning problems are wicked problems', in Cross N (Ed): 'Developments in Design Methodology', Wiley, pp 135-144 (1984).

6 'For Your Information - A Study of Information Management and Systems in Acute Hospitals', The Audit Commission, HMSO Publications, London, UK (1995).

7 Rosleff F: 'European Healthcare Trends: Towards Managed Care in Europe', Coopers \& Lybrand, London (1995).
8 Kennedy I: 'Learning from Bristol: the report of the public inquiry into children's heart surgery at the Bristol Royal Infirmary 1984 1995', Command Paper: CM 5207, The Stationery Office Limited (July 2001) - http://www.bristol-enquiry.org.uk/

9 Walker J, Pan E, Johnston D, Adler-Milstein J, Bates D W and Middleton B: 'The Value of Healthcare Information Exchange and Interoperability', Health Affairs, Web Exclusive (January 2005).

10 'eHealth - making health care better for European citizens', Commission of the European Communities, SEC(2004)539 (April 2004) - http://europa.eu.int/information_society/doc/qualif/ health/

11 'Computer Technology in Medical Education and Assessment', Background Report, Office of Technology Assessment, Congress of the United States, Library of Congress, (1979).

12 Ingram D: 'The Good European Health Record', in Laires M, Ladeira $M$ and Christensen J (Eds): 'Health in the New Communications Age', IOS Press, Amsterdam, pp 66-74 (1995).

13 Grimson J, Grimson W, Berry D, Stephens G, Felton E, Kalra D, Toussaint $P$ and Weier $O \mathrm{~W}$ : 'A CORBA-based integration of distributed electronic healthcare records using the synapses approach', IEEE Trans Inf Technol Biomed, 2, No 3, pp 124-38 (September 1998).

14 Ingram D, Lloyd D, Beale T, Schloeffel P, Heard S and Kalra D: 'The openEHR Foundation', - http://www.openehr.org/

15 CHIME (and also for information on GEHR Project, Synapses Project, Synex Project, CLEF Project) - http://www. chime.ucl.ac.uk/

$16 \mathrm{APoGI}$ - http://www.chime.ucl.ac.uk/APoGI/ 


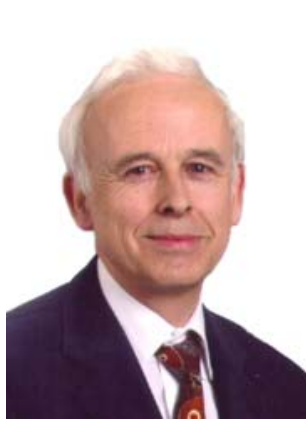

David Ingram is Director of the Centre for Health Informatics and Multiprofessional Education (CHIME) at University College London. After undergraduate Physics at Magdalen College Oxford, his career posts have been in industry, the National Health Service and University Medical Schools. CHIME is active in a wide range of international research programmes, and offers an extensive, multi-disciplinary educational programme, in quality, information and governance for health. The work of CHIME is founded on close working partnerships, with health service professionals, industry and community groups, and contributes to everyday services within the NHS and further afield.

His work in health informatics has covered contributions in the areas of mathematical modelling of biological and clinical systems, informatics in medical education, and formal methods for modelling health information requirements and architectures. Since 1990, he has been co-ordinating partner and partner in six major EU Health Informatics Framework Programme projects, focusing on health record architecture, implementation and standards, internationally. He is currently a principal investigator in the Medical Research Council e-Science Programme (Clinical e-Science Framework project, CLEF). He is active in research on representation of knowledge about inherited diseases. In 2003, CHIME was officially designated as a World Health Organisation Collaborating Centre, working on genetics knowledge management for patient advisory systems.

His professional activities include membership of the MRC Health and Bioinformatics Advisory Board, the national e-Science Advisory Board, the eScience Advisory Board of the Central Computing Laboratory for the Research Councils and the UK Council for Health Informatics Professions. He is the founder and chairman of the board of the openEHR Foundation, which is dedicated to sharing of standardised and open source software to support interoperable electronic health records. He is a Trustee of Starthere, an innovative charity which is establishing a national, highly accessible network of electronic signposts to local healthcare support services. He is international advisor to the EU project promoting health informatics in the accession states of the EU. He was elected Honorary Member of the Royal College of Physicians of London in 1999, in recognition of his contributions to medical science.

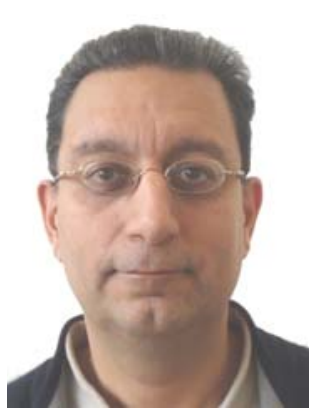

Dr Dipak Kalra is a clinical senior lecturer at CHIME (UCL) and a former GP. He plays a leading international role in research and development of HER interoperability architectures and prototyping and evaluation of new systems for health care records. His key research activities include a wide range EU funded Framework Programme projects, since 1991. He is leading both a European (CEN) standards Task Force and an ISO Project Team to develop an electronic health record communications (interoperability) standard. He is currently involved in an MRC e-Science project, CLEF, which is developing a pseudonymised repository of cancer records and a query workbench to support bio-informatics research. He co-ordinates the development and deployment of an NHS-accredited general practice computer system in north London. He was a GP Principal for eight years in a deprived part of east London and led the Medical Audit Advisory Group of that health authority for nine years. $\mathrm{He}$ is a Director of the openEHR Foundation and a member of its new Architecture Review Board. He is also an active member of HL7.

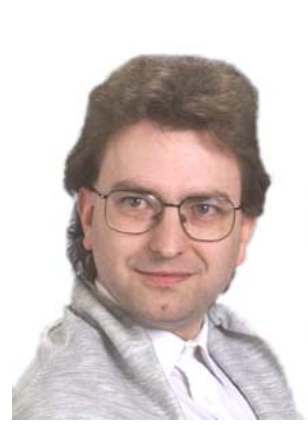

Originally a graduate of Computer Science with an emphasis on artificial intelligence methods, Tony Austin's career in Health Informatics began in decision support systems for the diagnosis and management of asthma, and the therapeutic control of anticoagulant drugs. More recently, he has designed and built a server for electronic healthcare records which has provided implementation experience for several standards tracks in the field, including CEN and ISO. Practical deployments of the server include the Department of Cardiovascular Medicine in a large London teaching hospital which has enabled him to continue his work in anticoagulant therapy. The server delivers real clinical benefits to thousands of Warfarin patients in this setting.

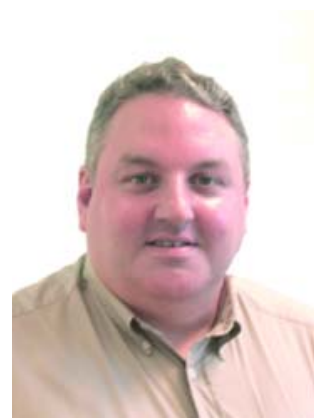

Matthew Darlison is a Senior Research Fellow in Clinical and Applied Bioinformatics at CHIME (UCL). Following training in German and comparative literature, he developed an interest in both theoretical and technical aspects of hypertext and the nascent World Wide Web in 1994. Since 1997 he has been technical lead of the APoGl project, researching and developing ways in which the Web can be used to supply information support to clinicians delivering services for haemoglobin disorders. He has also provided informatics support to a variety of the related projects, including the national patient registers, the CEGEN thalassaemia module and the development of standards for clinical care in thalassaemia.

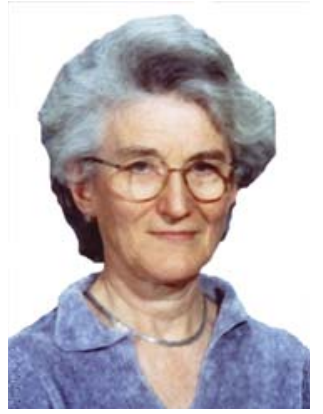

Bernadette Modell graduated in 1952 as a biologist and did her PhD in Cambridge when molecular biology was in its earliest exciting stages. She then studied medicine, and, after graduating, focused on management and prevention of haemoglobin disorders, as a model of genetics in the community.

As a Wellcome Principal Research Fellow she developed the basic scientific concepts of Community Genetics (a fusion of public health and primary healthcare aspects of medical genetics) in collaboration with WHO. She is retired in theory, but hardly in practice.

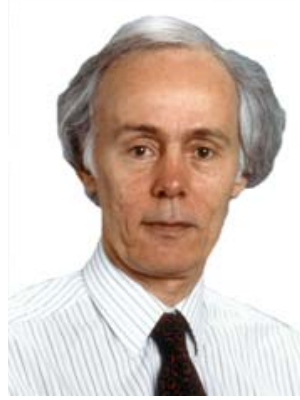

David Patterson is a Consultant Physician and Cardiologist based at the Whittington Hospital. He is also UCL Vice Dean and Campus Director of the Archway Campus.

He has had an interest in Health Informatics for some 20 years and has developed advisory and management systems for anticoagulant control which support the new models of service delivery.

He has also been involved in developing new roles for nurses and pharmacists in the delivery of these new service models. 